\title{
Sino-Himalayan mountains act as cradles of diversity and immigration centres in the diversification of parrotbills (Paradoxornithidae)
}

\author{
Yang Liu ${ }^{1}$, Junhua $\mathrm{Hu}^{2}$, Shou-Hsien $\mathrm{Li}^{3}$, Pablo Duchen ${ }^{4,5}$, Daniel \\ Wegmann $^{4,5}$ and Manuel Schweizer ${ }^{6 *}$
}

\footnotetext{
${ }^{1}$ State Key Laboratory of Biocontrol and College of Ecology and Evolution, Sun Yat-sen University, Guangzhou 510275, China,

${ }^{2}$ Chengdu Institute of Biology, Chinese Academy of Sciences, Chengdu 610041, China, ${ }^{3}$ Department of Life Science, National Taiwan Normal University, Taipei 116, Taiwan, ${ }^{4}$ Department of Biology, University of Fribourg, Chemin du Musée 10, CH 1700 Fribourg, Switzerland, ${ }^{5}$ Swiss Institute of Bioinformatics, 1700 Fribourg, Switzerland, ${ }^{6}$ Naturhistorisches Museum der Burgergemeinde Bern, Bernastrasse 15, CH 3005 Bern, Switzerland
}

\begin{abstract}
Aim Montane regions like the Sino-Himalayas constitute global diversity hotspots. Various mechanisms such as in situ adaptive divergence, speciation following immigration or allopatric diversification in complex landscapes have been proposed to account for the exceptional diversity found in a particular clade in a montane setting. We investigated macroevolutionary patterns to test these different hypotheses in the continental radiation of a Sino-Himalayan bird group, the parrotbills (Paradoxornithidae).
\end{abstract}

Location Sino-Himalayan region, Indo-Burma.

Methods We used phylogenetic comparative methods based on a multilocus, time-calibrated phylogeny to reconstruct patterns of lineage diversification, biogeographical history, morphological evolution as well as of climate niche history using ecological niche modelling.

Results The radiation of parrotbills started c. $12 \mathrm{Ma}$, diversifying at an apparent constant rate over time. The biogeographical history appears to be complex, within-region speciation in mountains was restricted to China. Size evolution was concentrated in the early phase of parrotbill radiation, whereas morphological shape evolution did not differ from Brownian motion. We found no indication for niche conservatism, with climate niche evolution occurring throughout the radiation of parrotbills.

Conclusions Parrotbills diversified within a time span of increased regional orogenesis and associated strong climate change. While the south-west and central Chinese mountains were revealed to be a species pump, with in situ allopatric diversification triggered by complex topography and high habitat turnover, the diversity in the Himalayas was chiefly the result of immigration. Evidence for continuous ecological specialization and for the absence of climate niche conservatism could be interpreted as the consequence of ongoing climate- and habitat-induced ecological opportunities. The radiation of parrotbills demonstrates the influence of multiple drivers of diversification in a single group due to the dynamic geological and palaeoclimatic history of the SinoHimalayan region and illustrates the complex nature of continental radiations.

\section{Keywords}

biogeographical history, climate niche evolution, continental radiation, diversification, ecological niche modelling, ecological opportunity, morphological evolution, parrotbills, Passeriformes, Sino-Himalayan region 


\section{INTRODUCTION}

Mountain ranges harbour exceptionally high levels of biodiversity and often constitute hotspots of species endemism, especially in tropical and subtropical regions (e.g. Myers et al., 2000). Various evolutionary and ecological processes have been proposed to explain such exceptional diversity. Montane regions not only provide many opportunities for geographical isolation followed by allopatric speciation (e.g. sister species occurring at the same elevations on different mountain tops), but also present ample possibilities for adaptive diversification ('in situ diversification hypothesis') along altitudinal gradients (Doebeli \& Dieckmann, 2003; Korner, 2007). In addition, the immigration of pre-adapted lineages with subsequent speciation ('immigration hypothesis') within a mountain range has often been found to be an important driver of diversification in montane species assemblages (Johansson et al., 2007; Price et al., 2014).

These different processes of species accumulation are, however, not always mutually exclusive, and may occur simultaneously or subsequently in a group of organisms. Moreover, independent of the process involved, both abiotic and biotic factors, such as climate, topography and interspecific competition and their interactions, as well as geometric constraints, influence montane species richness patterns (e.g. Colwell et al., 2004; Wu et al., 2013; Srinivasan et al., 2014; Hughes \& Atchison, 2015). Therefore, disentangling different historical processes and assessing the relative importance of drivers shaping diversity patterns is of primary importance in understanding the underlying mechanisms of global mountain biodiversity.

One major montane hotspot for global biodiversity is located in the Sino-Himalayan region, i.e. the Himalayas and the adjacent sub-Himalayan region of Indo-Burma as well as the south-western and central Chinese mountain ranges (hereafter, Himalayan/SW/C Chinese mountains) (Myers et al., 2000; Päckert et al., 2012; Lei et al., 2015). Studies on the diversification of species-rich clades in this region, based on molecular phylogenies have revealed incongruent patterns, which might at least partly be the result of the different ecological requirements of the groups studied (Favre et al., 2015). In situ diversification triggered by habitat fragmentation and climate oscillation associated with the uplift of the Qinghai-Tibetan Plateau, for instance, have been suggested for several groups of plants (e.g. Wen et al., 2014; Zhang et al., 2014) and insects (e.g. Condamine et al., 2012; Schmidt et al., 2012). Similarly, the high diversity of bird species in the SW/C Chinese mountains has been suggested to be the result of in situ diversification in an area of high topographic complexity and long-term environmental stability (Wu et al., 2013; Qu et al., 2014; Lei et al., 2015).

In contrast, in situ divergence and speciation along altitudinal gradients seem to have been of little importance in some clades of birds. Indeed, comprehensive studies on two passerine groups, namely Old World leaf warblers
(Phylloscopidae) and the superfamily Corvoidea, as well as on a complete songbird (Passeriformes) community of the East Himalayas demonstrated that the diversity in the Himalayas was largely caused by immigration, especially from central China and Southeast Asia, and that the evolution of body size and foraging modes preceded the establishment of altitudinal range differences (Johansson et al., 2007; Kennedy et al., 2012; Price et al., 2014). Elevation parapatry seems to have been largely caused by the immigration of boreal avifaunal elements from the north-east, whereas relatively few (sub)tropical species adapted successfully to temperate habitats (Päckert et al., 2012). Although ecological opportunity generated by mountain orogenesis and associated climate and habitat changes facilitated immigration and hence species build-up in the Himalayas, it did not directly drive speciation as in classical models of adaptive radiation (cf. Johansson et al., 2007).

Such contrasting results illustrate the complexity of the build-up of Sino-Himalayan biota. To obtain a broader picture of the relevant mechanisms and processes, more comparative studies are needed using integrative approaches combining species' evolutionary histories with ecological traits and niche characteristics (cf. Hughes \& Atchison, 2015). A particularly conspicuous group to study in this respect are the parrotbills (Paradoxornithidae), a passerine family of the Sino-Himalayan regions comprising around 20 species (Robson, 2007). The monophyly of this group was recently corroborated by a comprehensive molecular phylogenetic study, although the position of the Nearctic wrentit Chamaea fasciata was controversial (Moyle, 2005). Parrotbills have the highest diversity in the East Himalayas and SW/C Chinese mountains, with up to 12 species in central Sichuan (Fig. 1, Robson, 2007), but their distribution extends to the northern part of Indo-Burma in the south and the Palaearctic region of East Asia in the north. The different species occur in a variety of habitats, ranging from bamboo scrubs, reed-beds and grasslands to broadleaf and coniferous forests; the clade comprises high-altitude specialists (up to $3500 \mathrm{~m}$ ) as well as mid and low elevation species, and small-range endemics as well as species with a wide distribution area (Robson, 2007). Parrotbills share a parrot-like thick and sturdy bill, however, the exact shape and particular size of the bill vary among species. Importantly, they also vary considerably in body size (Robson, 2007), and as both bill shape and body size are functional traits well known to be under adaptive selection in other bird groups (Grant \& Grant, 2002), parrotbills are likely candidates for ecological adaptations in montane regions. As an indicative example, bill-size is significantly correlated with trophic niche width in the generalist vinous-throated parrotbill (Paradoxornis webbianus) (Hsu et al., 2014). Overall, this diverse morphology in combination with complex distribution patterns and habitat variation suggest that the different parrotbill species have adapted to a broad array of ecological niches, which makes them ideal for investigating drivers of diversification in a montane setting. 


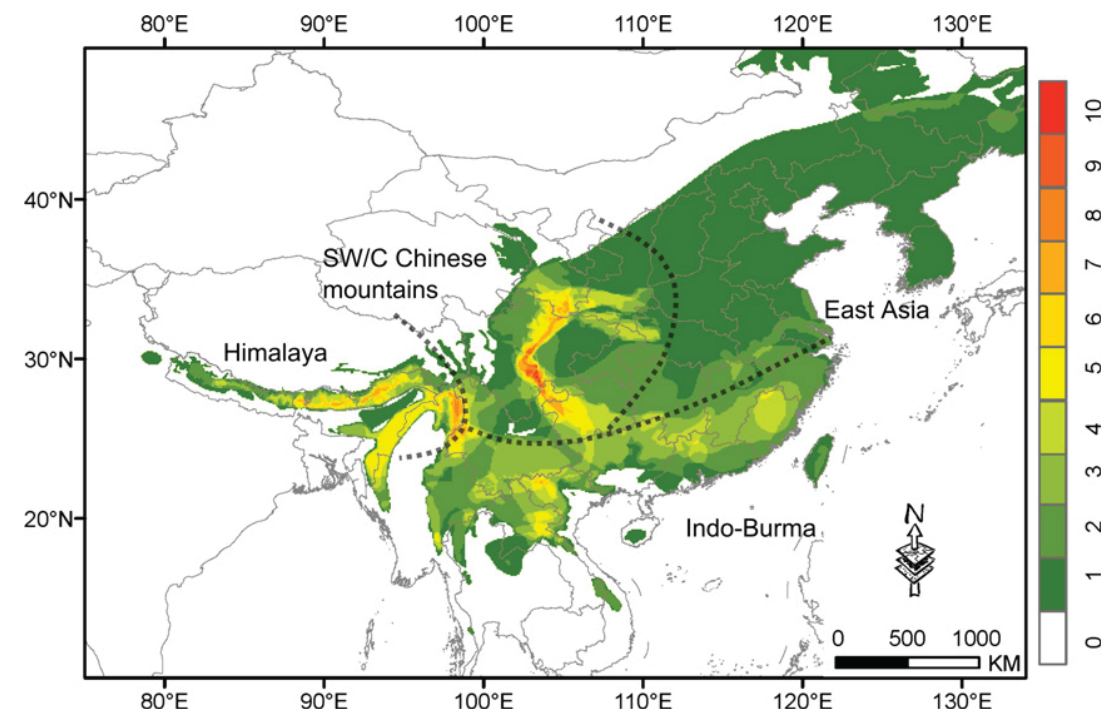

Figure 1 Diversity of the passerine family Paradoxornithidae, parrotbills. Species richness was computed by overlapping range maps of each species from the Bird Species Distribution Maps of the World (BirdLife International and NatureServe, 2014) using ArcGIS 9.2 (ESRI, Redland, CA). Warm colours indicate areas with high species diversity, whereas cold colours indicate sites with few species.
Here, we aim at contrasting two alternative scenarios to explain the pattern of species richness of parrotbills in the Himalayas and central Chinese mountains. In the first scenario, immigration followed by subsequent allopatric diversification is assumed to be the major driver for the pattern of diversity in this region, as has been found for several bird taxa and in an entire bird community in the Himalayas (Johansson et al., 2007; Kennedy et al., 2012; Päckert et al., 2012; Price et al., 2014). In this scenario, we expect that the sister taxa of Himalayan and central Chinese species occur outside these mountain systems. In other words, adaptive divergence is expected to have occurred outside the Himalayan/central Chinese hotspot. Immigration into this region might then have been facilitated by ecological opportunity generated by documented climate change 6-10 million years ago (Ma) (cf. Johansson et al., 2007; Price, 2008). As a consequence, ecological differences and associated morphological adaptations would have evolved early in the clade's history and would be partitioned between rather than within subclades, and sister species would merely be ecological replacements of each other.

In the alternative scenario, the diversity of parrotbills in the Himalayan/SW/C Chinese mountain hotspot is the result of in situ diversification. This could either have been the result of allopatric speciation across barriers caused by complex topography or by adaptive divergence, especially along altitudinal gradients, and their associated environmental parameters (reviewed in Favre et al., 2015). In the latter case, ecological and associated morphological differentiations are expected to have occurred within subclade radiations endemic to different mountain regions. Thus, sister species co-occurring in the same region should differ substantially in ecology and morphology. Variation in these parameters is consequently expected within rather than among subclades. If, on the other hand, allopatric speciation due to complex topography was the main driver of in situ diversification, we would expect sister species occurring in the same region to be morphologically and ecologically similar, with most variation found between rather than within subclades.

To test these alternative scenarios, we used phylogenetic comparative methods based on a time-calibrated multilocus molecular phylogeny and comprehensive morphological data in combination with ecological niche analyses and a biogeographical reconstruction of diversification events.

\section{MATERIALS AND METHODS}

\section{Phylogenetic analyses}

To infer a species tree, we used the multispecies coalescent algorithm * $*_{\text {BEAST }}$ as implemented in BEAST 1.7 .5 (Drummond \& Rambaut, 2007; Heled \& Drummond, 2010) based on two mitochondrial genes, two autosomal nuclear markers and one Z-linked marker from 18 of 20 extant species retrieved from Yeung et al. (2011), implementing published substitution rates for calibration (see Appendix S1, Table S1 in Supporting Information).

\section{Lineage diversification}

The R packages 'Laser' 2.4.1 (Rabosky, 2006b), 'Geiger' 2.0.1 (Harmon et al., 2008) and 'Phytools' 0.9-93 (Revell, 2012) were used for all comparative data modelling. Temporal variation in diversification rates within parrotbills was visualized with semi-logarithmic lineage-through-time plots. We tested whether diversification rates have changed over time, fitting different likelihood models for diversification rates on the maximum clade credibility species tree from the * BEAST analyses and comparing their Akaike's information criterion (AIC) scores (Rabosky, 2006a; Rabosky \& Lovette, 2008) (see Appendix S1). An increase in model fit was generally considered to be significant when the reduction in AIC score in a more complex model was $\geq 2$ (Burnham \& Anderson, 2002). 


\section{Morphological evolution}

We measured the length from bill to skull, defined as the distance from the angle at the front of the skull to the tip (BSk), bill width at feathering and bill depth at the highest point of the bill (Bmax), as well as tail and tarsus length from museum specimens of several individuals and subspecies per species (see Table S2). Morphological data were gathered for all species for which genetic data were available except Paradoxornis flavirostris. Natural-log (ln) transformed mean trait values were then used for further analyses.

The multivariate ration analysis of Baur \& Leuenberger (2011) was used to obtain an overall measure of size (isosize) for each taxon and size-independent principal components in terms of ratios. The 'PCA ratio spectrum' and 'allometry ratio spectrum' (Baur \& Leuenberger, 2011) were applied to interpret the principal components in shape space (shape PC1 and shape PC2).

Mean subclade disparity-through-time (DTT) (Harmon et al., 2003) was then calculated for isosize as well as for shape PC1 and shape PC2, using average squared Euclidean distances among species traits and the species tree. Observed values were compared with a null distribution from 10,000 simulations of Brownian motion trait evolution over our species tree. The area between the DTT plot for the data and the mean of the simulations (MDI, morphological disparity index) were calculated. Moreover, we calculated the MDI value between each simulation of trait evolution under a Brownian motion pro- cess and our own data and then determined the proportion of cases with an MDI value greater/smaller than or equal to zero (cf. Slater et al., 2010).

We additionally fitted six likelihood models of continuous morphological evolution to our data and compared their AIC scores, based on the species tree (Table 1; see Appendix S1).

\section{Biogeographical reconstruction}

The $\mathrm{R}$ package 'BioGeoBears' 0.2.1 (Matzke, 2013b) was used for biogeographic reconstruction based on the species tree. According to their current distribution, species were assigned to one or more of the following biogeographical regions: Himalayas, SW/C Chinese mountains, Indo-Burma, East Asia. The Mekong-Salween divide was used as the border between Himalayas and SW/C Chinese mountains and the southern provinces of China north to the Yangtze were treated as part of Indo-Burmese region (cf. Johansson et al., 2007; Li et al., 2011; Päckert et al., 2012). Our definition of the SW/C Chinese mountains basically included the Southwest Mountain Region and the western and northern part of Central China defined by $\mathrm{Qu}$ et al. (2014). The maximum range size was set to three, as no extant species occurs in more than three biogeographical regions. Dispersal was restricted to adjacent areas. We compared the following six models of biogeographical reconstruction in the

Table 1 Different models of continuous morphological evolution fitted to the time-calibrated species tree with parameter and values.

\begin{tabular}{|c|c|c|c|c|c|c|}
\hline & Beta & Parameters & AIC & $\Delta \mathrm{AIC}$ & $\mathrm{AICc}$ & $\Delta \mathrm{AICc}$ \\
\hline \multicolumn{7}{|l|}{ Isosize } \\
\hline $\mathrm{BM}$ & 0.003 & & -15.977 & 2.435 & -15.12 & 1.446 \\
\hline $\mathrm{OU}$ & 0.003 & alpha $=0$ & -13.977 & 4.435 & -12.131 & 4.435 \\
\hline TDL & 0.008 & $b=-0.074$ & -17.563 & 0.849 & -15.717 & 0.849 \\
\hline $\mathrm{EB}$ & 0.015 & $r=-0.232$ & -18.412 & 0 & -16.566 & 0 \\
\hline DDL & 0.032 & $\mathrm{~b}=-0.002$ & -3.621 & 14.791 & -1.621 & 14.945 \\
\hline DDX & 0.043 & $r=-0.022$ & -5.849 & 12.563 & -3.849 & 12.717 \\
\hline \multicolumn{7}{|c|}{ Shape PC1 } \\
\hline $\mathrm{BM}$ & 0.007 & & -3.67 & 1.884 & -2.813 & 0.741 \\
\hline $\mathrm{OU}$ & 0.018 & alpha $=0.214$ & -4.776 & 0.778 & -2.93 & 0.624 \\
\hline TDL & 0 & $b=-0.0003$ & -3.772 & 1.781 & -1.772 & 1.782 \\
\hline EB & 0.007 & $r=-0.000001$ & -1.67 & 3.884 & 0.176 & 3.73 \\
\hline DDL & 0.019 & $b=-0.0004$ & -5.554 & 0 & -3.554 & 0 \\
\hline DDX & 0.019 & $r=-0.001$ & -5.545 & 0.009 & -3.545 & 0.009 \\
\hline \multicolumn{7}{|c|}{ Shape PC2 } \\
\hline $\mathrm{BM}$ & 0.001 & & -33.745 & 0 & -32.888 & 0 \\
\hline $\mathrm{OU}$ & 0.001 & alpha $=0$ & -31.745 & 2 & -29.899 & 2.989 \\
\hline TDL & 0.001 & $b=-0.008$ & -31.754 & 1.992 & -29.907 & 2.981 \\
\hline $\mathrm{EB}$ & 0.001 & $r=-0.012$ & -31.758 & 1.987 & -29.912 & 2.976 \\
\hline DDL & 0.008 & $b=-0.0005$ & -26.962 & 6.784 & -24.962 & 7.926 \\
\hline DDX & 0.008 & $r=-0.010$ & -26.155 & 7.59 & -24.155 & 8.733 \\
\hline
\end{tabular}

BM, Brownian motion; OU, Ornstein-Uhlenbeck; TDL, time-dependent linear model with linear rate change through time; EB, time-dependent early-burst model with exponential rate change through time; DDL, diversity-dependent linear model with rate changing linearly with changing species diversity; DDX, diversity-dependent linear model with rate changing exponentially with changing species diversity. Beta, rate of morphological evolution at the basal node of the phylogeny; alpha, bounding parameter for the OU models; b, slope in the TDL and DDL model; $r$, parameter in the EB and DDX models. 


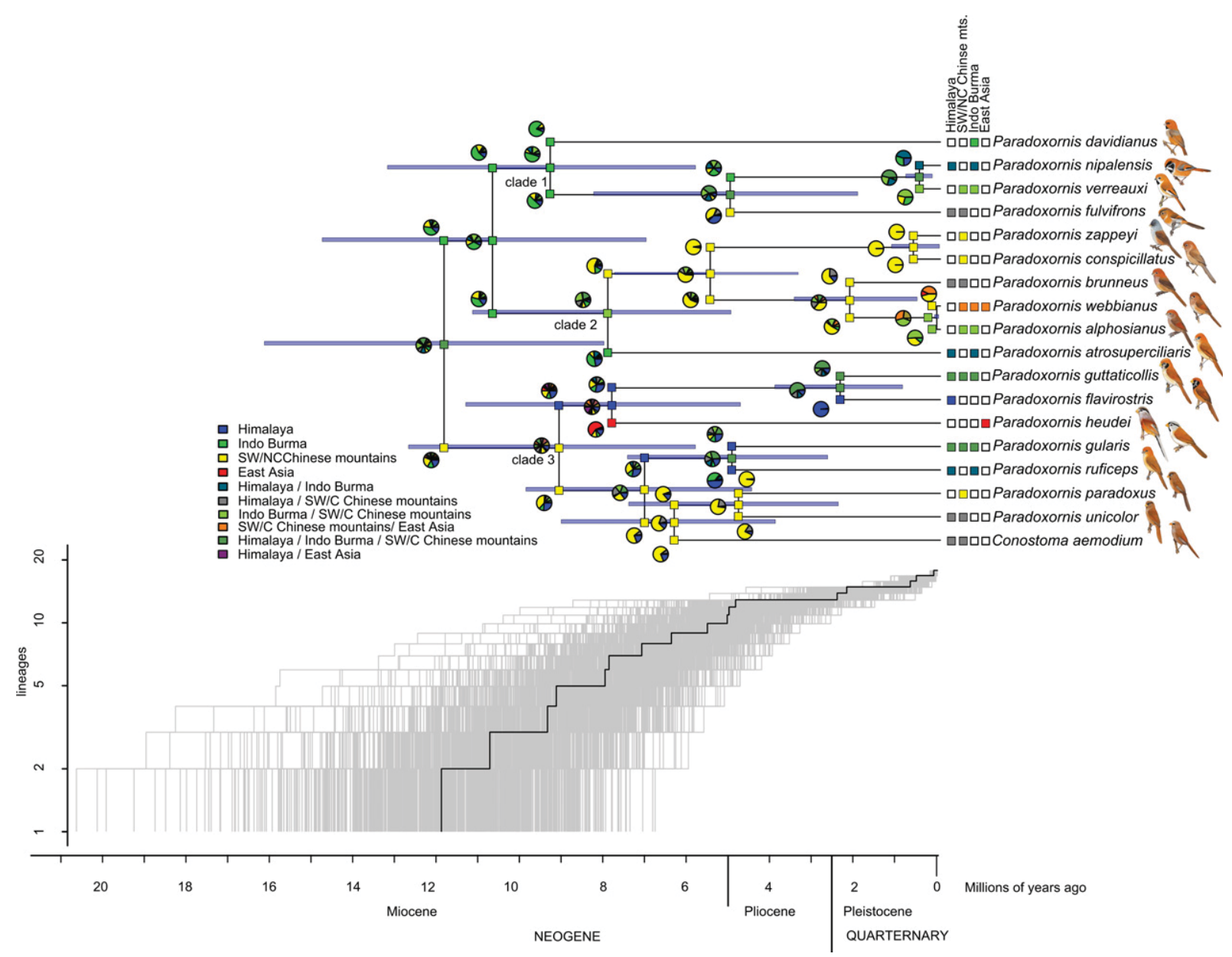

Figure 2 Maximum clade credibility species tree (top) of parrotbills, biogeographical reconstruction based on a dispersal-extinction cladogenesis model (DEC) and semi-logarithmic lineages-through-time (LTT) plots (bottom). Mean node ages and the 95\% highest posterior density distributions are shown (blue bars). Moreover, the distribution of terminal taxa is given. The lower part of the figure displays semi-logarithmic LTT plots for 1000 random trees from the posterior distribution in grey and for the maximum clade credibility tree in black. Parrotbill figures are used with permission from Robson (2007).

Table 2 Comparison of the fit of different models of biogeographical range evolution and model specific estimates for the different parameters $[d=$ dispersal, $e=$ extinction, $j=$ weight of jump dispersal (founder speciation) $]$.

\begin{tabular}{|c|c|c|c|c|c|c|c|c|c|}
\hline Model & $\operatorname{Ln} L$ & Parameter nb & $d$ & $e$ & $j$ & AIC & $\Delta \mathrm{AIC}$ & $\mathrm{AICc}$ & $\triangle \mathrm{AICc}$ \\
\hline DEC & -50.71 & 2 & 0.06 & $1.49 \times 10^{-02}$ & 0 & 105.4 & 0 & 106.2 & 0 \\
\hline $\mathrm{DEC}+\mathrm{J}$ & -49.89 & 3 & 0.05 & $1.00 \times 10^{-12}$ & 0.09 & 105.8 & 0.4 & 107.5 & 1.3 \\
\hline DIVALIKE & -51.69 & 2 & 0.07 & $1.00 \times 10^{-12}$ & 0 & 107.4 & 2 & 108.2 & 2.0 \\
\hline DIVALIKE $+\mathrm{J}$ & -51.13 & 3 & 0.06 & $1.00 \times 10^{-12}$ & 0.08 & 108.3 & 2.9 & 110.0 & 3.8 \\
\hline BAYAREALIKE & -53 & 2 & 0.06 & $1.01 \times 10^{-01}$ & 0 & 110 & 4.6 & 110.8 & 4.6 \\
\hline BAYAREALIKE $+\mathrm{J}$ & -52.79 & 3 & 0.06 & $9.27 \times 10^{-02}$ & 0.03 & 111.6 & 6.2 & 113.3 & 7.1 \\
\hline
\end{tabular}

Figure 3 Evolution of shape PCs and isosize along lineages (top) and disparity-through-time (DTT) plot for shape PCs, isosize and mid-point elevation (bottom) of parrotbills. Species trees are shown with colours of branches indicating reconstructed evolution of the three morphological parameters along lineages. Bars at tips are proportional to parameter values of extant species. In the DTT plot, the solid line indicates the mean subclade DTT based on the species tree, while the dashed line represents the median expected subclade disparity derived from 10,000 simulations under Brownian motion evolution. The area shaded in grey indicates the $95 \%$ range for the simulated data. 


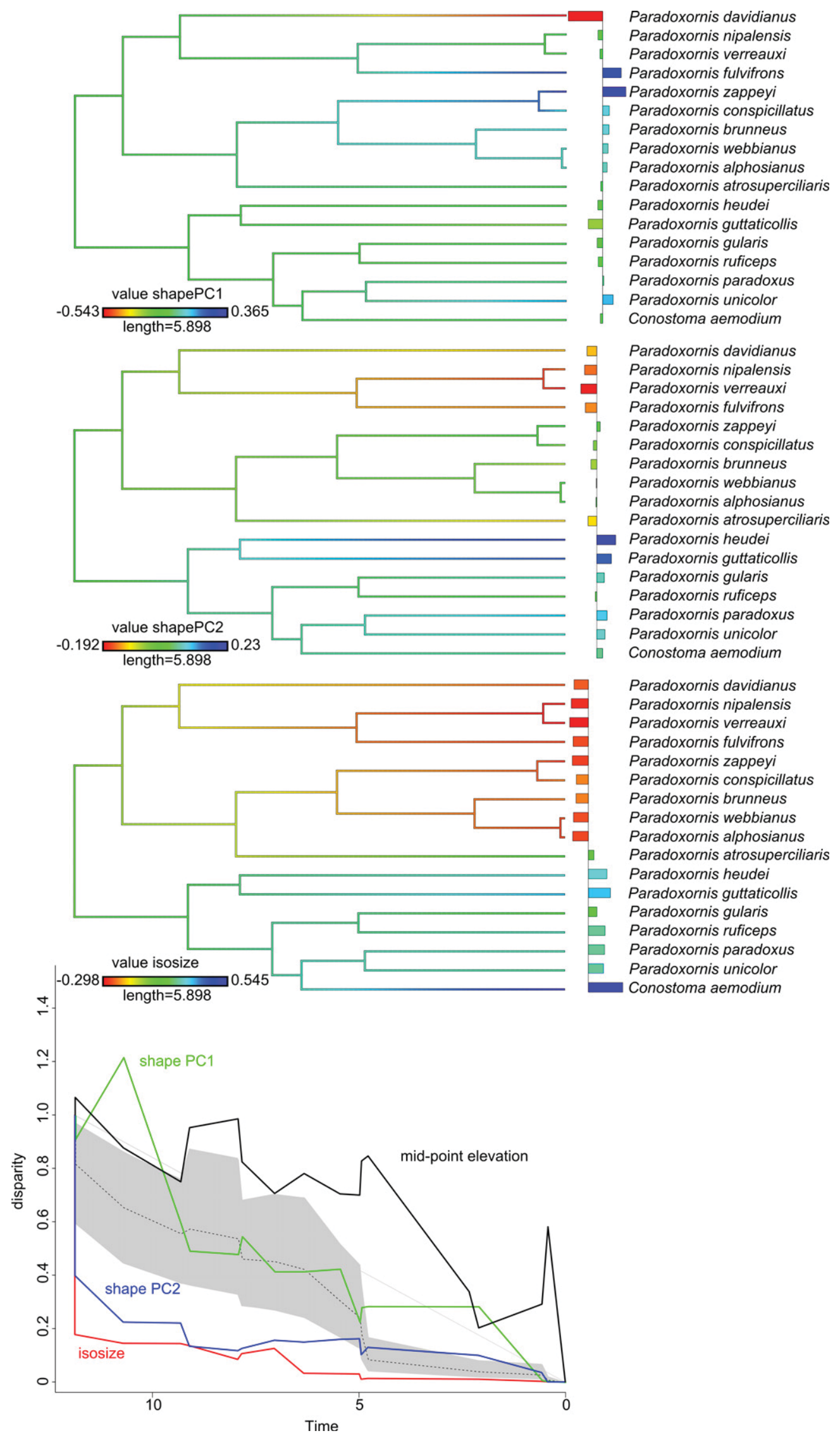


likelihood framework, as implemented in 'BioGeoBears': dispersal-extinction cladogenesis model (DEC) (Ree et al., 2005), dispersal-vicariance analysis (DIVA) (Ronquist, 1997) and the BayArea model (Landis et al., 2013), plus all three models separately under the possibility of founder events (+J) (Matzke, 2013a, 2014). The fit for the different models was assessed using the AIC scores.

\section{Ecological niche modelling and niche overlaps and equivalency}

We carried out ecological niche modelling (ENM) using Maxent 3.3.3k (Phillips \& Dudik, 2008; Hu \& Liu, 2014) based on 19 bioclimatic variables and occurrence data from different sources (see Appendix S2).

To evaluate the niche overlap between species, we calculated two indices, i.e. Schoener's $D$ and $I$ (Warren et al., 2008) for each species pair. These values measured the similarity of projected suitability for each grid cell of the study area and ranged from 0 (no niche overlap) to 1 (complete overlap). We tested the null hypothesis of niche identity in ENMTools 1.3 (Warren et al., 2008, 2010).

\section{Reconstruction of predicted niche occupancy and disparity in a phylogenetic context}

The potential distributions of each species derived from the ENMs were combined in order to assess the evolution of climatic niches among species, using the R package 'PHyLoclim' (Heibl \& Calenge, 2013). Following the approach of Evans et al. (2009), we computed predicted niche occupancy (PNO) profiles. The occurrence probability of each species in each grid cell of the study area from the MAxENT model was combined with the corresponding scores of the original bioclimatic variables to create a vector of probabilities per binned score (100 intervals).

To reconstruct the evolution of predicted niche occupancy, the maximum likelihood estimate of the bioclimatic variables at each of the internal nodes of the species tree was assessed under the assumption of Brownian motion evolution (Evans et al., 2009). This process was repeated using 100 random samples on the basis of niche occupancy profiles for each of the extant taxa.

We used the mean predicted climate occupancy for each species and each bioclimatic variable to calculate mean subclade DTT, using squared Euclidean distances among species and the species tree as described above for morphology. Mean subclade DTT was also calculated for mid-point elevation.

Finally, we used Schoener's $D$ and $I$ to test for phylogenetic signals in patterns of niche overlap, using an age-range correlation (Fitzpatrick \& Turelli, 2006). The significance of the correlation was assessed via 10,000 Monte Carlo simulations by random permutations with the niche-overlap matrix to estimate the distribution of slope and intercept based on the null hypothesis of no phylogenetic signal.

\section{RESULTS}

\section{Phylogenetic relationships}

The species tree revealed basically three clades: clade 1, consisting of small-sized species with complex head patterns; clade 2 , chiefly comprising small species with a uniform colour pattern; clade 3, consisting of large-sized species (Fig. 2). All clades were robustly supported with posterior probabilities $>0.95$, with the exception of the sister group relationship between clades 1 and 2 , which had a posterior probability of 0.81 . The tree topology was overall congruent with the results of Yeung et al. (2011).

The diversification of parrotbills started c. $12 \mathrm{Ma}$, with the three clades emerging c. 9-8 Ma. Most speciation events occurred c. 8-5 Ma.

\section{Lineage diversification}

We found no strong indication for a major shift in diversification rates in parrotbills (see Appendix S1, Table S3). As simulations suggested considerable power to detect rate shifts in trees of the size relevant here (18 tips) when changes in rate were at least 10 -fold (see Fig. S1), we concluded that diversification rates were either constant or have changed only subtly over the course of evolution in this group.

\section{Morphological evolution}

Subclade disparity for isosize was significantly lower than expected under Brownian motion, with an MDI of -0.258 $(P=0$; Fig. 3$)$. Different size classes were partitioned early among the different clades, with clade 1 and 2 consisting of small species except for $P$. atrosuperciliaris in clade 2, and clade 3 consisting of the larger species. In accordance with this result, a time-dependent early-burst model was found to be the best-fitting model of continuous evolution for isosize (Table 1), although, it was only significantly different from Brownian motion evolution when considering $\triangle \mathrm{AIC}$, but not for $\triangle \mathrm{AICc}$.

Shape PC1 was dominated by the ratio of tail length and depth of bill (see Figs S2 \& S3). Subclade disparity was high at the beginning, influenced by the short-tailed taxon $P$. davidianus, followed by lower values and, again, values clearly above the expected values under Brownian motion (dashed line) from $c .5 \mathrm{Ma}$ onwards. It was consequently overall significantly higher than expected under Brownian motion, with an MDI of $0.132(P=0.009)$. According to the reconstruction of morphological evolution along lineages, differentiation in shape PC1 occurred mainly between 7 and $4 \mathrm{Ma}$ (Fig. 3). A diversity-dependent linear model was found to be the best-fitting model of continuous evolution for shape PC1 (Table 1), however, it was not significantly different from Brownian motion evolution. 
Shape PC2 was dominated by the ratio of width of bill and length of bill (see Figs S2 \& S3). Subclade disparity was significantly lower than expected under Brownian motion, with an MDI of $-0.180(P=0.0001$; Fig. 3$)$. Different bill shapes were thus partitioned early among subclades. Nonetheless, Brownian motion was found to be the best-fitting model of continuous trait evolution (Table 1).

\section{Biogeographical reconstruction}

A DEC was found to be the best-fitting model (Table 2). Ancestral areas and area splits could not be inferred for all nodes with high probability (Fig. 3). While no ancestral area or area combination could be revealed with high probability for the ancestral node, the origin of clade 1 most probably was Indo-Burma, while the radiation of $C$. aemodium, $P$. unicolor and $P$. paradoxus mostly took place in the SW/C Chinese mountains, with subsequent colonization of the Himalayas in two of the tip taxa. After the split of $P$. atrosuperciliaris, the radiation in the remaining taxa of clade 2 also happened in the SW/C Chinese mountains, with colonization of all other regions by the tip taxa. The only split in tip taxa that was restricted to a single biogeographical region was between $P$. zappeyi and P. conspicillatus in the SW/C Chinese mountains (Fig. 3).

\section{Ecological niche modelling, niche overlap and equivalency}

We assessed the discrimination abilities of the ENMs by computing the area under the receiver operating characteristic curve (AUC) (details in Appendix S2). Across all parrotbill species, the average values of training $(70 \%$ of the occurrences for model training) and testing (the remaining 30\% data for testing the resulting models) AUC ranged between 0.82 and 0.99 , indicating enough discrimination abilities (see Fig. S4). Visual comparisons of the ENMs showed strong species-level variations, mostly in accordance with the available maps of species-specific distributions (see Fig. S5).

In total, 84 out of 136 comparisons (61.8\%) between taxa resulted in $D$ being smaller than the threshold of 0.4 defined by Rödder \& Engler (2011). The high overlap values (e.g. $D>0.6$ ) were observed in only 11 pairs (6.7\%; Table 3$)$. Further, the niche equivalency hypothesis was rejected for 229 out of 272 species comparisons (84.2\%; $P<0.05$; see Table 3), indicating that niches of species pairs were significantly distinct in most cases. We detected no significant phylogenetic signal for either $I$ or $D$ when applying the age-range correlation.

The reconstruction of niche occupancy through time revealed overlapping internal nodes, indicating convergent climatic origins, especially for annual mean temperature, temperature seasonality, annual precipitation and precipitation seasonality (Fig. 4). Crossing branches for all variables indicated that convergent climatic niche evolution among clades was prevalent throughout parrotbill radiation and some sister taxa displayed clear differences for some variables.

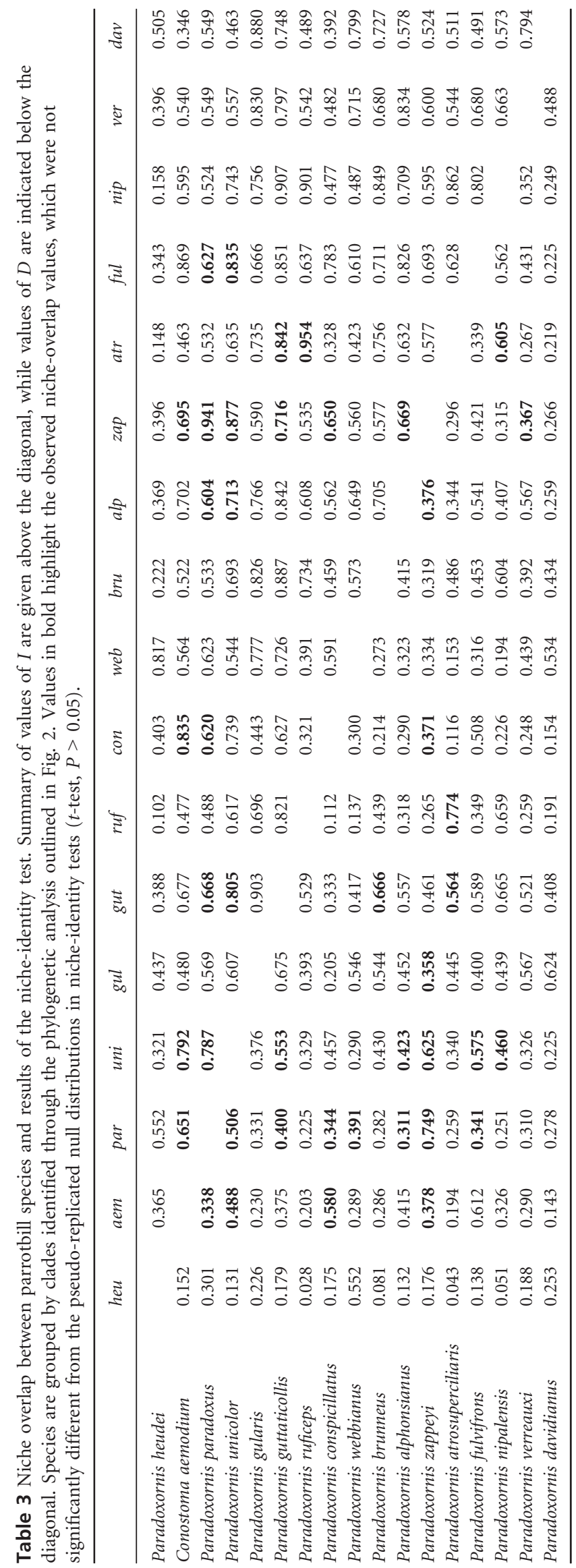



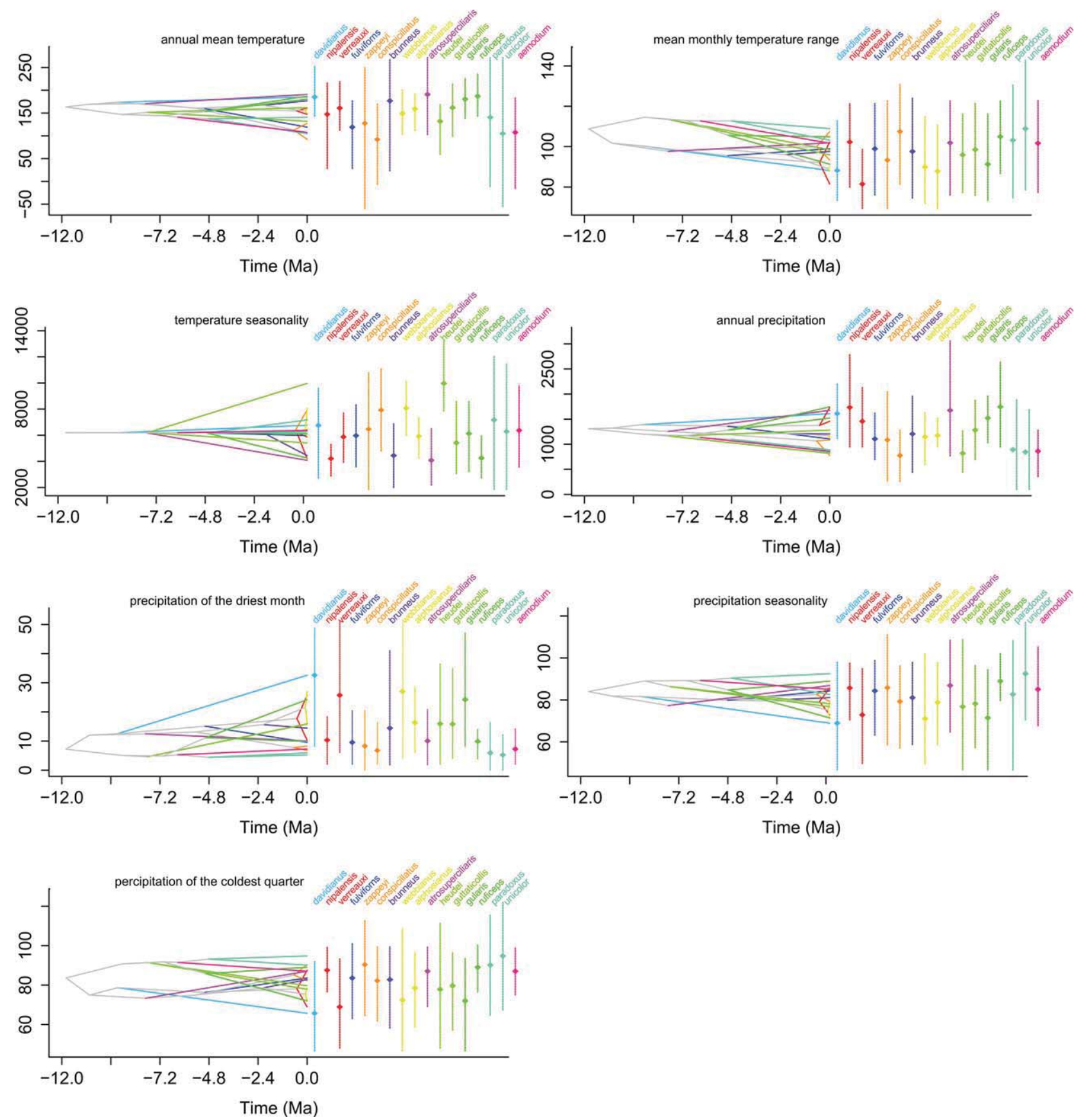

Figure 4 History of the evolution of predicted climate niche for the seven bioclimatic variables and each species based on the species tree of parrotbills. This process was repeated using 100 random samples on the basis of niche occupancy profiles for each extant taxa. The vertical dashed line indicates the $80 \%$ central density of climate tolerance and the mean is indicated by a point. Different colours refer to different clades. Temperature data are in ${ }^{\circ} \mathrm{C} * 10$.

Accordingly, subclade disparity was usually higher than expected under Brownian motion for all climate variables and increased towards the present for all variables except for annual mean temperature and annual precipitation (Fig. 5). Consequently, MDIs were positive for all climate variables. For mid-point elevation, disparity within subclades was found to be higher than among subclades throughout the radiation of parrotbills (Fig. 3).

\section{DISCUSSION}

\section{Spatio-temporal diversification patterns}

The diversification of parrotbills falls within a time span of increased regional orogenesis and associated strong climate change. Although the uplift in the Himalayan region began as early as $50 \mathrm{Ma}$, significant orogenesis occurred from 10 to 
Figure 5 Disparity-through-time (DTT) plot based on the mean predicted climate occupancy for each species and each of the seven relevant bioclimatic variables, illustrating the accumulation of relative ecological DTT. The solid line indicates the mean subclade DTT based on the species tree, while the dashed line represents the median expected subclade disparity derived from 10,000 simulations under Brownian motion evolution. The area shaded in grey indicates the $95 \%$ range for the simulated data.

$8 \mathrm{Ma}$ and even more recently (Zhisheng et al., 2001). Major climate shift occurred between 10-6 Ma in association with the onset of the Indian and East Asian monsoon (Johansson et al., 2007; Price et al., 2014). In the SW/C Chinese mountains (e.g. Hengduan mountains and mountains around the Sichuan Basin) intensive tectonic activities and environmental changes then followed at the Mio-Pliocene boundary (Song et al., 2009; Qu et al., 2014; Wu et al., 2014).

Ecological competition for resources, geographical aspects or failure to keep pace with a changing environment might limit diversification (Moen \& Morlon, 2014), which is apparently the case when considering whole communities or larger clades of birds in the Himalayas or part of this mountain range (Kennedy et al., 2012; Price et al., 2014). As we found no evidence for a slowdown in diversification, however, such processes seem not to be prevalent in clades such as the parrotbills, whose distribution areas span larger areas such as continents or several biogeographical (sub)regions. In congruence, no slowdown in diversification rate was detected in continental bird radiations in the Neotropics (Derryberry et al., 2011; Schweizer et al., 2014).
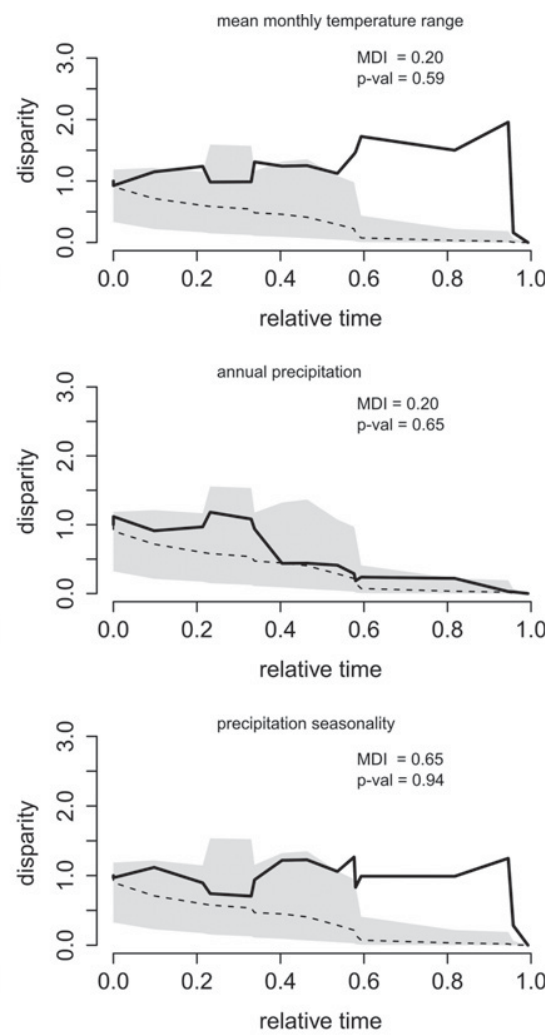

relative time

lative time

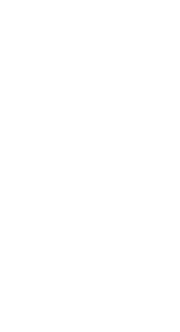

The SW/C Chinese Mountain system was revealed to be the centre of diversification and source for the colonization into the remaining biogeographical regions for at least two clades (within clades 2 and 3; Fig. 2). These radiations started in the late Miocene and resulted in more than half of the current parrotbill species richness (10 out of 18 species). The SW/C Chinese Mountain system is characterized by high local species replacement and high beta diversity (Lei et al., 2015). This striking pattern is thought to be the result of both evolutionary and ecological isolation due to highly diversified habitats and subregional geographical isolation due to complex topography (Lei et al., 2015; Qu et al., 2015). The only speciation event among terminal taxa within a single region also occurred in the SW/C Chinese Mountain system and concerned P. conspicillatus and P. zappeyi. The timeframe of their split comprised the early Pleistocene glaciation period. During this period, glaciers, which were restricted to high altitudes in the central Chinese mountains, acted as dispersal barriers between the remaining stable habitats at lower elevations, leading to local isolation ( $\mathrm{Qu}$ et al., 2014, 2015). P. conspicillatus and P. zappeyi display no clear climate niche differences, but might show some ecological 
differentiation, with P. zappeyi being restricted to open forests and scrub patches on exposed peaks and ridges near mountain tops (Robson, 2007). Both are similar in size and shape PC2, but differ slightly in shape PC1 (see above). Nonetheless, we have no indication that parapatric speciation along an altitudinal gradient played a role.

On the other hand, we found no indication that the Himalayas were a centre of diversification or in situ speciation. The high diversity especially found in the Eastern Himalayas is thus the result of dispersal from either SW/C China or Indo-Burma into that region with or without subsequent speciation. This is consistent with the results of other studies, which revealed that the Himalayan avifaunal species assemblage is chiefly the result of immigration with virtually no in situ speciation (Johansson et al., 2007; Päckert et al., 2012; Price et al., 2014). The only species whose distribution is restricted to the Himalayan region is P. flavirostris. This species is a specialist of grasslands in floodplains and not a montane species (Robson, 2007). The East Himalayas are most species-rich, with declining richness towards the west, reflecting a commonly found pattern in birds and mammals (e.g. Price et al., 2011; Srinivasan et al., 2014). Unlike the SW/C Chinese Mountains, most of the Himalayas were covered by ice or dominated by alpine vegetation during the Pleistocene. The Eastern Himalayas, however, remained vegetated and might have acted as a refuge for many taxa during glacial maxima (cf. Srinivasan et al., 2014). Most of the colonization events of the Himalayas in parrotbills seem to have happened before the Pleistocene. Their distribution might have been restricted to suitable habitat in the east of the mountain chain during glacial periods from where a subset of species could have extended their ranges towards the west when more favourable conditions were prevalent. However, this hypothesis can only be tested formally in further studies using population level sampling.

\section{Morphological diversification}

We found an indication that the evolution of size in parrotbills was rapid early in their evolution, followed by a slowdown. Climate change in combination with ongoing orogenesis was prevalent in the early diversification of parrotbills (see above) and might have generated ecological opportunities that promoted ecological diversification in terms of niche dimensions for which body size matters. A similar pattern was found in another continental bird radiation (Schweizer et al., 2014), but also in the vangas (Vangidae) endemic to Madagascar (Jønsson et al., 2012) and in several other bird lineages (Harmon et al., 2010), which mirrors a pattern expected in a classical adaptive radiation process (Harmon et al., 2003).

Bill shape is a strong predictor for feeding specializations in birds (Grant \& Grant, 2002) and slenderness of bill might be a proxy for trophic characters of food in P. webbianus (Hsu et al., 2014). Especially shape PC2, but also shape PC1, were found to be influenced by bill measurements. Only for shape
PC2, did we find an indication that its evolution slowed down through time. This suggests that although bill shape evolution and corresponding ecological specialization might have experienced a burst in the early phase of parrotbill radiation, it continued throughout their diversification, leading to further ecological differentiations within size classes.

\section{Climate niches}

When inferring climate niche divergence between parrotbills, we found strong inter-specific variation in potential climatic niches, with few species pairs holding high overlap values. Accordingly, we also rejected the hypothesis that species pairs were distributed in identical climate niches by means of niche-identity tests in most cases. This is congruent with the expectation that closely related species are generally not identical in their extant climate niches (Warren et al., 2014), and can be interpreted as evidence for niche divergence across extant parrotbill species. In general, sister species seem to occupy rather different climate niches, although some might also be similar for some bioclimatic variables. In agreement with this, it was recently shown that even the most recently diverged species of Paradoxornithidae, $P$. webbianus and $P$. alphosianus, occupy different climate niches (Shaner et al., 2015).

Moreover, climate niche evolution seems to have occurred throughout the radiation of parrotbills and hence we have no indication for the prevalence of climate niche conservatism. It is difficult to discuss these results in a comparative way, as studies on the evolution of climate niche tolerances in continental radiations of birds are scarce. Similar to our results, however, a tendency towards divergent climate niche evolution within clades and convergent evolutions among clades was revealed in South and South-East Asian Pomatorhinus scimitar babblers (Nyari \& Reddy, 2013).

In parrotbills, the lack of climate conservatism might be a consequence of ecological opportunities generated by climate and associated habitat change throughout their radiation. To be able to survive through major climate changes, different species might have survived in suitable microclimatic pockets (cf. Hof et al., 2011). This might be the case for small-range endemics in the SW Chinese mountain systems such as $P$. zappeyi, P. brunneus or P. conspicillatus. However, a broad climatic tolerance might also have resulted in a similar pattern and have facilitated parrotbills in coping with ongoing climate change. Some parrotbill species are indeed known to have rather broad realized niches, with, for example, $P$. webbianus being a diet and habitat generalist occurring from sea level to c. $2600 \mathrm{~m}$ (Robson, 2007; Hsu et al., 2014).

\section{CONCLUSIONS}

Although the Himalayas and the adjacent sub-Himalayan region of Indo-Burma as well as the south-western and central Chinese mountain ranges harbour a high level of avian 
diversity, few studies have used phylogenetic comparative methods to test alternative hypotheses for the observed diversity patterns. To our knowledge, this is the first comprehensive study to use a comparative analytical framework integrating phylogenetic, biogeographical, morphological and climate niche data to infer macroevolutionary patterns in a continental radiation of birds. We found that speciation in parrotbills occurred over a rather large time period; hence, different processes, such as in situ diversification and speciation following immigration, have seemingly played a role. Evidence for continuous morphological evolution corresponding to ecological specialization and for a lack of climate niche conservatism might be interpreted as the consequence of ongoing climate- and habitat-induced ecological opportunities. Collectively, this study demonstrates the influence of multiple drivers of diversification in a single group due to the dynamic geological and palaeoclimatic history of the Sino-Himalayan region, and illustrates the complex nature of continental radiations.

\section{ACKNOWLEDGEMENTS}

We are grateful to Yun Li, Guoling Chen, Lu Liang, Xueyan Li for their help in retrieving published sequences and online occurrence data; to Peng He [Institute of Zoology, Chinese Academy of Sciences (CAS)], Luming Liu (Kunming Institute of Zoology, CAS), Binglan Zhang (the BioMuseum, Sun Yat-sen University), Zhaohe Tang (Fujian Normal University) for providing access to their specimen collections; also to Josep del Hoyo and Lynx Edicions for the use of paintings of parrotbills from the Handbook of the Birds of the World. We further thank Stefan T. Hertwig and Lukas Rüber for commenting on the manuscript and Heather Murray for language editing. This research is supported by the National Natural Science Foundation of China (No. 31301875 and 31270568), Youth Innovation Promotion Association CAS (2015304), and the Specimen Platform of China (2005DKA 21403-JK), and by a grant from the Swiss National Science Foundation (No. 31003A_149920) to D.W.

\section{REFERENCES}

Baur, H. \& Leuenberger, C. (2011) Analysis of ratios in multivariate morphometry. Systematic Biology, 60, 813825.

BirdLife International and NatureServe (2014) Bird species distribution maps of the world. BirdLife International and NatureServe, Cambridge.

Burnham, K.P. \& Anderson, D.R. (2002) Model selection and mulitimodel inference, 2nd edn. Springer-Verlag, New York.

Colwell, R.K., Rahbek, C. \& Gotelli, N.J. (2004) The middomain effect and species richness patterns: what have we learned so far? The American Naturalist, 163, E1-E23.

Condamine, F.L., Sperling, F.A.H., Wahlberg, N., Rasplus, J.Y. \& Kergoat, G.J. (2012) What causes latitudinal gradi- ents in species diversity? Evolutionary processes and ecological constraints on swallowtail biodiversity. Ecology Letters, 15, 267-277.

Derryberry, E.P., Claramunt, S., Derryberry, G., Chesser, R.T., Cracraft, J., Aleixo, A., Pérez-Emán, J., Remsen, J.J.V. \& Brumfield, R.T. (2011) Lineage diversification and morphological evolution in a large-scale continental radiation: the Neotropical ovenbirds and woodcreepers (Aves: Furnariidae). Evolution, 65, 2973-2986.

Doebeli, M. \& Dieckmann, U. (2003) Speciation along environmental gradients. Nature, 421, 259-264.

Drummond, A.J. \& Rambaut, A. (2007) BeAst: Bayesian evolutionary analysis by sampling trees. BMC Evolutionary Biology, 7, 214.

Evans, M.E.K., Smith, S.A., Flynn, R.S. \& Donoghue, M.J. (2009) Climate, niche evolution, and diversification of the 'bird-cage' evening primroses (Oenothera, sections Anogra and Kleinia). The American Naturalist, 173, 225-240.

Favre, A., Päckert, M., Pauls, S.U., Jähnig, S.C., Uhl, D., Michalak, I. \& Muellner-Riehl, A.N. (2015) The role of the uplift of the Qinghai-Tibetan Plateau for the evolution of Tibetan biotas. Biological Reviews, 90, 236-253.

Fitzpatrick, B.M. \& Turelli, M. (2006) The geography of mammalian speciation: mixed signals from phylogenies and range maps. Evolution, 60, 601-615.

Grant, P.R. \& Grant, B.R. (2002) Unpredictable evolution in a 30-year study of Darwin's finches. Science, 296, 707-711.

Harmon, L.J., Schulte, J.A., Larson, A. \& Losos, J.B. (2003) Tempo and mode of evolutionary radiation in iguanian lizards. Science, 301, 961-964.

Harmon, L.J., Weir, J.T., Brock, C.D., Glor, R.E. \& Challenger, W. (2008) Geiger: investigating evolutionary radiations. Bioinformatics, 24, 129-131.

Harmon, L.J., Losos, J.B., Davies, T.J., Gillespie, R.G., Gittleman, J.L., Jennings, W.B., Kozak, K.H., McPeek, M.A., Moreno-Roark, F., Near, T.J., Purvis, A., Ricklefs, R.E., Schluter, D., Schulte, J.A., Seehausen, O., Sidlauskas, B.L., Torres-Carvajal, O., Weir, J.T. \& Mooers, A.O. (2010) Early bursts of body size and shape evolution are rare in comparative data. Evolution, 64, 2385-2396.

Heibl, C. \& Calenge, C. (2013) Phyloclim: integrating phylogenetics and climatic niche modelling. (http://CRAN. R-project.org/package= phyloclim)

Heled, J. \& Drummond, A.J. (2010) Bayesian inference of species trees from multilocus data. Molecular Biology and Evolution, 27, 570-580.

Hof, C., Levinsky, I., Araújo, M.B. \& Rahbek, C. (2011) Rethinking species' ability to cope with rapid climate change. Global Change Biology, 17, 2987-2990.

Hsu, Y.-C., Shaner, P.-J., Chang, C.-I., Ke, L. \& Kao, S.-J. (2014) Trophic niche width increases with bill-size variation in a generalist passerine: a test of niche variation hypothesis. Journal of Animal Ecology, 83, 450-459.

Hu, J.H. \& Liu, Y. (2014) Unveiling the conservation biogeography of a data-deficient endangered bird species under climate change. PLoS ONE, 9, e84529. 
Hughes, C.E. \& Atchison, G.W. (2015) The ubiquity of Alpine plant radiations: from the Andes to the Hengduan Mountains. New Phytologist, 207, 275-282.

Johansson, U.S., Alström, P., Olsson, U., Ericson, P.G.R., Sundberg, P. \& Price, T.D. (2007) Build-up of the Himalayan avifauna through immigration: a biogeographical analysis of the Phylloscopus and Seicercus warblers. Evolution, 61, 324-333.

Jønsson, K.A., Fabre, P.H., Fritz, S.A., Etienne, R.S., Ricklefs, R.E., Jorgensen, T.B., Fjeldså, J., Rahbek, C., Ericson, P.G.P., Woog, F., Pasquet, E. \& Irestedt, M. (2012) Ecological and evolutionary determinants for the adaptive radiation of the Madagascan vangas. Proceedings of the National Academy of Sciences USA, 109, 6620-6625.

Kennedy, J.D., Weir, J.T., Hooper, D.M., Tietze, D.T., Martens, J. \& Price, T.D. (2012) Ecological limits on diversification of the Himalayan core Corvoidea. Evolution, 66, 2599-2613.

Korner, C. (2007) The use of 'altitude' in ecological research. Trends in Ecology and Evolution, 22, 569-574.

Landis, M.J., Matzke, N.J., Moore, B.R. \& Huelsenbeck, J.P. (2013) Bayesian analysis of biogeography when the number of areas is large. Systematic Biology, 62, 789-804.

Lei, F., Qu, Y., Song, G., Alström, P. \& Fjeldså, J. (2015) The potential drivers in forming avian biodiversity hotspots in the East Himalaya-Mountains of Southwest China. Integrative Zoology, 10, 171-181.

Li, Y., Zhai, S.N., Qiu, Y.X., Guo, Y.P., Ge, X.J. \& Comes, H.P. (2011) Glacial survival east and west of the 'MekongSalween Divide' in the Himalaya-Hengduan Mountains region as revealed by AFLPs and cpDNA sequence variation in Sinopodophyllum hexandrum (Berberidaceae). Molecular Phylogenetics and Evolution, 59, 412-424.

Matzke, N.J. (2013a) Probabilistic historical biogeography: new models for founder-event speciation, imperfect detection, and fossils allow improved accuracy and model-testing. Frontiers of Biogeography, 5, 242-248.

Matzke, N.J. (2013b) BioGeoBEARS: BioGeography with Bayesian (and Likelihood) evolutionary analysis in $\mathrm{R}$ Scripts. R package, version 0.2.1, published 27 July 2013. Available at: http://CRAN.R-project.org/package=BioGeoBEARS.

Matzke, N.J. (2014) Model selection in historical biogeography reveals that founder-event speciation is a crucial process in island clades. Systematic Biology, 63, 951970.

Moen, D. \& Morlon, H. (2014) Why does diversification slow down? Trends in Ecology and Evolution, 29, 190-197.

Moyle, R.G. (2005) Phylogeny and biogeographical history of Trogoniformes, a pantropical bird order. Biological Journal of the Linnean Society, 84, 725-738.

Myers, N., Mittermeier, R.A., Mittermeier, C.G., da Fonseca, G.A.B. \& Kent, J. (2000) Biodiversity hotspots for conservation priorities. Nature, 403, 853-858.

Nyari, A.S. \& Reddy, S. (2013) Comparative phyloclimatic analysis and evolution of ecological niches in the scimitar babblers (Aves: Timaliidae: Pomatorhinus). PLoS ONE, 8, e55629.

Päckert, M., Martens, J., Sun, Y.H., Severinghaus, L.L., Nazarenko, A.A., Ting, J., Töpfer, T. \& Tietze, D.T. (2012) Horizontal and elevational phylogeographic patterns of Himalayan and Southeast Asian forest passerines (Aves: Passeriformes). Journal of Biogeography, 39, 556573.

Phillips, S.J. \& Dudik, M. (2008) Modeling of species distributions with MAXent: new extensions and a comprehensive evaluation. Ecography, 31, 161-175.

Price, T. (2008) Speciation in birds. Roberts and Company, Greenwood Village, CO.

Price, T.D., Mohan, D., Tietze, D.T., Hooper, D.M., Orme, C.D. \& Rasmussen, P.C. (2011) Determinants of northerly range limits along the Himalayan bird diversity gradient. The American Naturalist, 178(Suppl. 1), S97S108.

Price, T.D., Hooper, D.M., Buchanan, C.D., Johansson, U.S., Tietze, D.T., Alström, P., Olsson, U., Ghosh-Harihar, M., Ishtiaq, F., Gupta, S.K., Martens, J., Harr, B., Singh, P. \& Mohan, D. (2014) Niche filling slows the diversification of Himalayan songbirds. Nature, 509, 222-225.

Qu, Y.H., Ericson, P.G.P., Quan, Q., Song, G., Zhang, R.Y., Gao, B. \& Lei, F.M. (2014) Long-term isolation and stability explain high genetic diversity in the Eastern Himalaya. Molecular Ecology, 23, 705-720.

Qu, Y.H., Song, G., Gao, B., Quan, Q., Ericson, P.G.P. \& Lei, F.M. (2015) The influence of geological events on the endemism of East Asian birds studied through comparative phylogeography. Journal of Biogeography, 42, 179-192.

Rabosky, D.L. (2006a) Likelihood methods for detecting temporal shifts in diversification rates. Evolution, 60, 11521164.

Rabosky, D.L. (2006b) LASER: a maximum likelihood toolkit for detecting temporal shifts in diversification rates from molecular phylogenies. Evolutionary Bioinformatics Online, 2, 257-260.

Rabosky, D.L. \& Lovette, I.J. (2008) Explosive evolutionary radiations: decreasing speciation or increasing extinction through time? Evolution, 62, 1866-1875.

Ree, R.H., Moore, B.R., Webb, C.O. \& Donoghue, M.J. (2005) A likelihood framework for inferring the evolution of geographic range on phylogenetic trees. Evolution, 59, 2299-2311.

Revell, L.J. (2012) Рнутоols: an R package for phylogenetic comparative biology (and other things). Methods in Ecology and Evolution, 3, 217-223.

Robson, C. (2007) Familiy Paradoxornithidae (Parrotbills). Handbook of the birds of the world (ed. by J. del Hoyo, A. Elliott, J. Sargatal and D.A. Christie), pp. 292-321. Lynx Edicions, Barcelona.

Rödder, D. \& Engler, J.O. (2011) Quantitative metrics of overlaps in Grinnellian niches: advances and possible drawbacks. Global Ecology and Biogeography, 20, 915927. 
Ronquist, F. (1997) Dispersal-vicariance analysis: a new approach to the quantification of historical biogeography. Systematic Biology, 46, 195-203.

Schmidt, J., Opgenoorth, L., Holl, S. \& Bastrop, R. (2012) Into the Himalayan exile: the phylogeography of the ground Beetle Ethira clade supports the Tibetan origin of forest-dwelling Himalayan species groups. PLoS ONE, 7, e45482.

Schweizer, M., Hertwig, S.T. \& Seehausen, O. (2014) Diversity versus disparity and the role of ecological opportunity in a continental bird radiation. Journal of Biogeography, 41, 1301-1312.

Shaner, P.J.L., Tsao, T.H., Lin, R.C., Liang, W., Yeh, C.F., Yang, X.J., Lei, F.M., Zhou, F., Yang, C.C., Hung, L.M., Hsu, Y.C. \& Li, S.H. (2015) Climate niche differentiation between two passerines despite ongoing gene flow. Journal of Animal Ecology, 84, 829-839.

Slater, G.J., Price, S.A., Santini, F. \& Alfaro, M.E. (2010) Diversity versus disparity and the radiation of modern cetaceans. Proceedings of the Royal Society B: Biological Sciences, 277, 3097-3104.

Song, G., Qu, Y.H., Yin, Z.H., Li, S.S., Liu, N.F. \& Lei, F.M. (2009) Phylogeography of the Alcippe morrisonia (Aves: Timaliidae): long population history beyond late Pleistocene glaciations. BMC Evolutionary Biology, 9, 143.

Srinivasan, U., Tamma, K. \& Ramakrishnan, U. (2014) Past climate and species ecology drive nested species richness patterns along an east-west axis in the Himalaya. Global Ecology and Biogeography, 23, 52-60.

Warren, D.L., Glor, R.E. \& Turelli, M. (2008) Environmental niche equivalency versus conservatism: quantitative approaches to niche evolution. Evolution, 62, 2868-2883.

Warren, D.L., Glor, R.E. \& Turelli, M. (2010) ENMTools: a toolbox for comparative studies of environmental niche models. Ecography, 33, 607-611.

Warren, D.L., Cardillo, M., Rosauer, D.F. \& Bolnick, D.I. (2014) Mistaking geography for biology: inferring processes from species distributions. Trends in Ecology and Evolution, 29, 572-580.

Wen, J., Zhang, J.-Q., Nie, Z.-L., Zhong, Y. \& Sun, H. (2014) Evolutionary diversifications of plants on the Qinghai-Tibetan Plateau. Frontiers in Genetics, 5, 4.

Wu, Y.J., Colwell, R.K., Rahbek, C., Zhang, C.L., Quan, Q., Wang, C.K. \& Lei, F.M. (2013) Explaining the species richness of birds along a subtropical elevational gradient in the Hengduan Mountains. Journal of Biogeography, 40, 2310-2323.
Wu, Y.J., Colwell, R.K., Han, N., Zhang, R., Wang, W., Quan, Q., Zhang, C., Song, G., Qu, Y.H. \& Lei, F.M. (2014) Understanding historical and current patterns of species richness of babblers along a 5000-m subtropical elevational gradient. Global Ecology and Biogeography, 23, 1167-1176.

Yeung, C.K.L., Lin, R.C., Lei, F.M., Robson, C., Hung, L.M., Liang, W., Zhou, F.S., Han, L.X., Li, S.H. \& Yang, X.J. (2011) Beyond a morphological paradox: complicated phylogenetic relationships of the parrotbills (Paradoxornithidae, Aves). Molecular Phylogenetics and Evolution, 61, 192-202.

Zhang, J.Q., Meng, S.Y., Allen, G.A., Wen, J. \& Rao, G.Y. (2014) Rapid radiation and dispersal out of the QinghaiTibetan Plateau of an alpine plant lineage Rhodiola (Crassulaceae). Molecular Phylogenetics and Evolution, 77, 147-158.

Zhisheng, A., Kutzbach, J.E., Prell, W.L. \& Porter, S.C. (2001) Evolution of Asian monsoons and phased uplift of the Himalaya-Tibetan Plateau since Late Miocene times. Nature, 411, 62-66.

\section{SUPPORTING INFORMATION}

Additional Supporting Information may be found in the online version of this article:

Appendix S1 Phylogenetic analyses and species sampling, patterns of lineage diversification through time.

Appendix S2 Ecological niche modelling and niche overlaps and equivalency.

Appendix S3 Tables S1-S3, Figures S1-S5. 